\title{
ANÁLISIS COMPARATIVO DE PACIENTES CON ESTENOSIS DEGENERATIVA LUMBAR PURA (EDLP) Y ESTENOSIS SECUNDARIA A ESPONDILOLISTESIS DEGENERATIVA LUMBAR (ELDL) TRATADOS QUIRÚRGICAMENTE EN EL PERÍODO DE 2008 A 2011 EN EL HOSPITAL METROPOLITANO DE QUITO-ECUADOR
}

\author{
ANÁLISE COMPARATIVA DE PACIENTES COM ESTENOSE DEGENERATIVA PURA LOMBAR \\ (EDPL) E ESTENOSE SECUNDÁRIA A ESPONDILOLISTESE LOMBAR DEGENERATIVA \\ LOMBAR (ELDL) TRATADOS CIRURGICAMENTE NO PERÍODO DE 2008 A 2011 \\ NO HOSPITAL METROPOLITANO DE QUITO, EQUADOR
COMPARATIVE ANALYSIS IN PATIENTS WITH PURE DEGENERATIVE LUMBAR STENOSIS
(EDLP) AND STENOSIS SECUNDARY TO DEGENERATIVE LUMBAR SPONDILOLISTHESIS
(ELDL) SURGICALLY TREATED BETWEEN 2008-2011 AT THE HOSPITAL METROPOLITANO, QUITO, ECUADOR.

Jaime Moyano', Edison Ahttr', Madelin Bilbao², Sebastián De la Torre ${ }^{3}$

\begin{abstract}
RESUMEN
Objetivo: Establecer si existen diferencias demográficas, de tratamiento y en resultados entre los grupos de EDLP y ELDL.Métodos: Estudio retrospectivo observacional de 76 pacientes con EDL tratados quirúrgicamente. Se analizan por separado los datos demográficos y la evolución del grupo 1 (EDLP, 53 pacientes) y del grupo 2 (ELDL, 23 pacientes). Resultados: Grupo 1: 51\% mujeres y 49\% varones. Edad promedio 56,8 años. Evolución preoperatoria 34 meses; Grupo 2: 78\% de mujeres y 22\% de varones. Edad promedio 63,4 años. Evolución preoperatoria 10 meses. Los dos grupos fueron intervenidos con la misma técnica, por el mismo equipo quirúrgico. El porcentaje de satisfacción postoperatoria fue mejor para el grupo 1 cuando el tiempo de evolución preoperatoria fue menor a 18 meses. En el grupo 2 los hallazgos no fueron significativos.Conclusión: La estenosis por ELDL tiene características de edad y sexo muy diferentes dela EDLP. La cirugía precoz presentó un mayor porcentaje de satisfacción.
\end{abstract}

Descriptores: Columna vertebral; Estenosis espinal/cirugía, Espondilolistesis/cirugía.

\section{RESUMO}

Objetivo: Determinar se existem diferenças demográficas, de tratamento e nos resultados entre os grupos EDLP e ELDL. Métodos: Estudo observacional retrospectivo de 76 pacientes com EDLP tratados cirurgicamente. Analisamos separadamente os dados demográficos e os resultados do grupo 1 (EDLP, 53 pacientes) e do grupo 2 (ELDL, 23 pacientes). Resultados: Grupo 1: 51\% mulheres e 49\% homens; média de idade, 56,8 anos. Evolução pré-operatória, 34 meses. Grupo 2: 78\% mulheres e 22\% homens; média de idade, 63,4 anos. Evolução pré-operatória, 10 meses. Os dois grupos foram operados com a mesma técnica e pela mesma equipe cirúrgica. A porcentagem de satisfação pós-operatória foi maior no Grupo 1 quando a evolução pré-operatória foi inferior a 18 meses. No Grupo 2, os resultados não foram significativos. Conclusão: Na estenose secundária a ELDL, a idade e distribução por gênero foram muito diferentes do encontrado na EDLP. A cirurgia precoce gerou uma maior porcentagem de satisfação.

Descritores: Coluna vertebral; Estenose espinal/cirurgia; Espondilolistese/cirurgia.

\begin{abstract}
Objective: Establish whether there are differences regarding demographics, treatment and outcomes between the groups EDLP and ELDL. Methods: Retrospective observational study of 76 patients with EDLP surgically treated. Demographic data and outcomes of Group 1 (EDLP, 53 patients) and Group 2 (ELDL, 23 patients) were analyzed separately. Results: Group 1: 51\% female; 49\% male. Mean age, 56.8 years. Pre-surgical development, 34 months. Group 2: 78\% female; 22\% male. Mean age, 63.4 years. Pre-surgical development, 10 months. Both groups were operated with the same technique and by the same surgical team. In Group 1, postoperative satisfaction was higher when the pre-surgical development was less than 18 months. In Group 2 there where no significant findings. Conclusion: In stenosis secondary to ELDL, age and gender distribution were very different from that found in EDLP. Early surgery led to a higher percentage of satisfaction.
\end{abstract}

Keywords: Spine; Spinal stenosis/surgery; Spondylolisthesis/surgery.

1. Médico Especialista en Cirugía de Columna, Hospital Metropolitano de Quito, Ecuador.

2. Médico Asistencial Instituto Cirugía Vertebral Hospital Metropolitano de Quito, Ecuador.

3. Médico Residente Ortopedia y Traumatología Universidad Internacional Ecuador, Hospital Metropolitano de Quito, Ecuador. 


\section{INTRODUCCION}

Durante los últimos veinte años se ha visto un incremento en la esperanza de vida de la población general, llegando al momento a ser 75 años en promedio para hombres y 85 años en mujeres. Siendo las patologías degenerativas vertebrales las más comunes en la práctica clínica ${ }^{1}$.

La estenosis degenerativa lumbar usualmente presenta sintomatología dentro de la quinta a la séptima década de vida. Aparentemente no existe una predominancia en cuanto al sexo².

Es importante recordar que la estenosis lumbar según lo describió Arnoldi et al. ${ }^{3}$, se puede dividir en dos tipos: congénita y degenerativa.

La estenosis degenerativa lumbar se describe como un síndrome clínico que afecta a los miembros inferiores causando claudicación neurológica a la marcha y dolor ciático inespecífico, que mejora a la flexión lumbar, puede estar asociada o no a lumbalgia ${ }^{4,5,6}$. En los estudios de imagen se evidencia una disminución del espacio disponible para las estructuras neurales y venas peridurales de la columna lumbar, en otras palabras es la disminución del diámetro del conducto raquídeo lumbar (Figura 1) que se produce a consecuencia de un proceso degenerativo que afecta al disco, articulaciones facetarías y ligamentos amarillos ${ }^{1,2,4,7}$.

La estenosis degenerativa lumbar secundaria a espondilolistesis tiene un cuadro clínico similar pero de evolución mas rápida y el nivel más afectado es L4-L5, considerándose mas bien una estenosis focal ${ }^{1}$. La fisiopatología es degenerativa en presencia de una alteración en la orientación de las facetas articulares (Figura 2). La mayor laxitud articular de la mujer puede ser otra causa determinante a la listesis, relacionada con la diferencia hormonal entre el sexo femenino y masculino ${ }^{1,6}$.

Dentro de la bibliografía generalmente se incluye a la espondilolistesis degenerativa lumbar dentro de las casuísticas de estenosis degenerativa lumbar a pesar de que las características demográficas, fisiopatologías y velocidad de empeoramiento del cuadro clínico son muy diferentes ${ }^{1,2,4-6}$.

El objetivo principal es establecer si existen realmente diferencias demográficas, de evolución, de tratamiento y en los resultados entre los dos grupos.

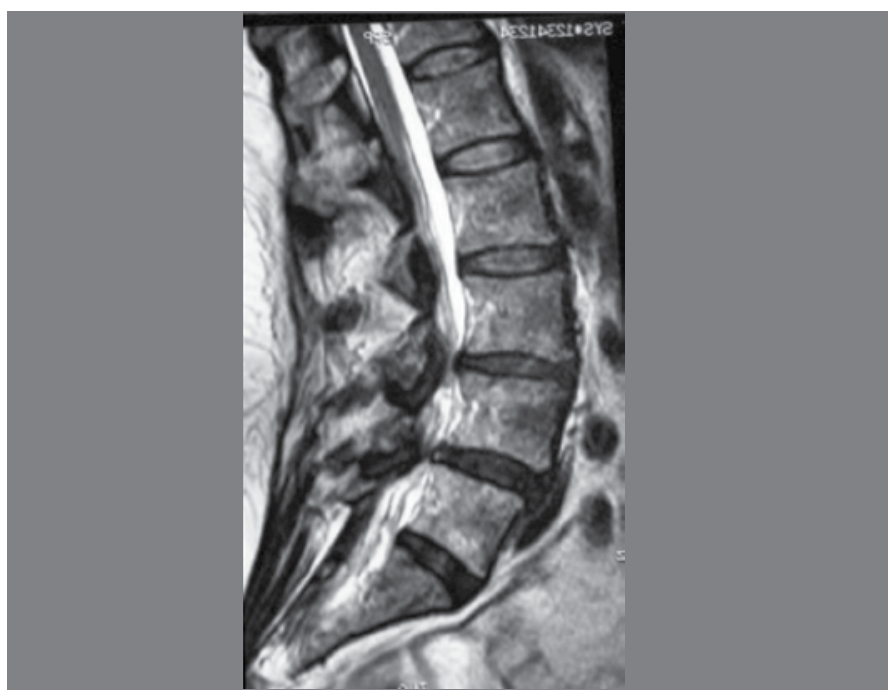

Figura 1: MRI Estenosis Pura L3-L4 y L4-L5.

\section{MATERIALES Y METODOS}

Es un estudio retrospectivo observacional, realizado en conjunto entre el Instituto de Cirugía Vertebral y el Servicio de Ortopedia y Traumatología del Hospital Metropolitano de Quito-Ecuador. Incluyó 76 pacientes tratados quirúrgicamente en el período comprendido entre enero del 2008 y enero del 2011, de los cuales 53 casos presentaban estenosis pura y 23 casos estenosis secundaria a espondilolistesis degenerativa.

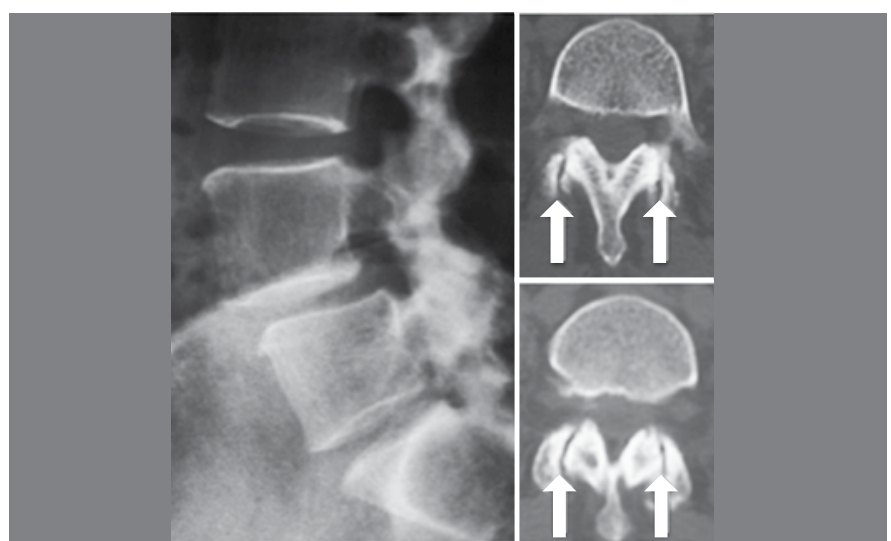

Figura 2. Rx Espondilolistesis degenerativa, alteración en la orientación de las facetas

Criterios de inclusión: Pacientes con historia clínica completa y una correlación clara entre la clínica y los estudios de imagen. Seguimiento mínimo de un año

Criterios de exclusión: Cirugía previa de columna vertebral, escoliosis, espondilolistesis traumática y seguimiento menor a un año.

Todos los pacientes fueron tratados por el mismo equipo quirúrgico. Se realizó en todos una descompresión lumbar amplia (Figura 3) por medio de laminectomía, foramenectomia, además se realizó una artrodesis transversa e instrumentación transpedicular (Figura 4).

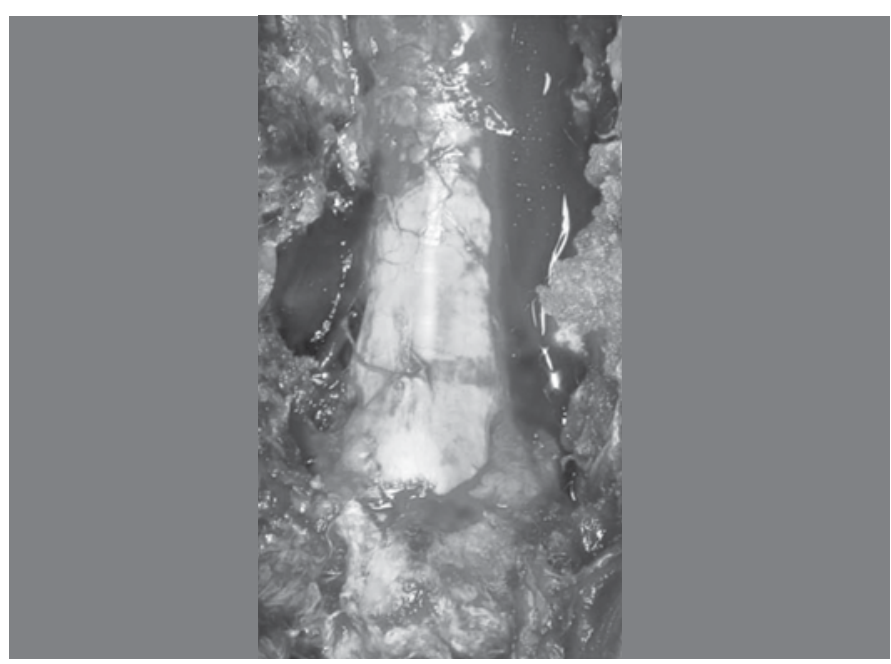

Figura 3. Laminectomia descompresiva ampliada.

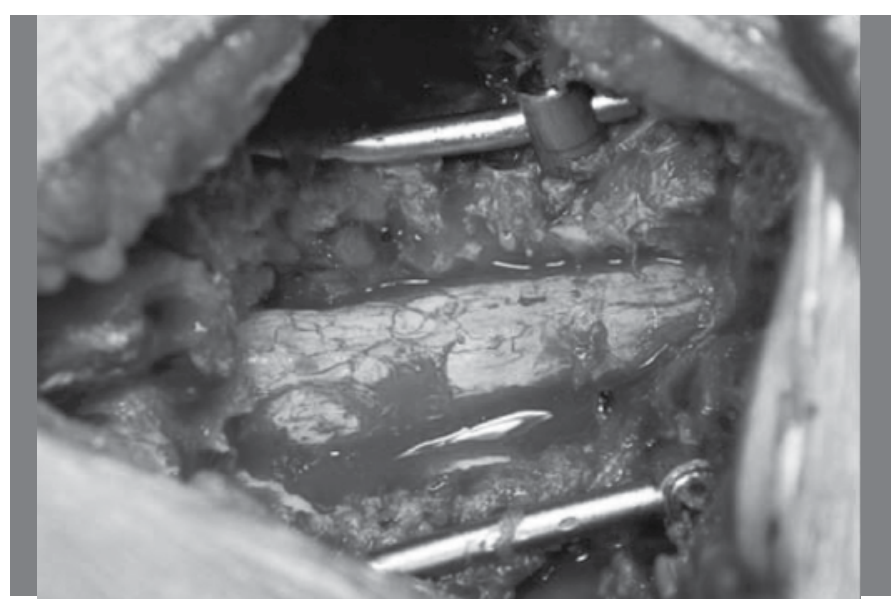

Figura 4. Instrumentación con tornillos transpediculares. 


\section{RESULTADOS}

En la distribución por género se encontró 26 casos de pacientes de sexo masculino (49\%) y 27 casos de sexo femenino (51\%) en el grupo de estenosis pura (Grupo 1). En el grupo de estenosis secundaria a espondilolistesis (Grupo 2) 5 pacientes fueron de sexo masculino (22\%) y 18 pacientes de sexo femenino (78\%) (Tabla 1 , Figuras 5 e 6$)$.

En relación a la edad, encontramos que en el Grupo 1 el rango de edad va desde 34 a 79 años, con un promedio de 56.8 años y en el Grupo 2 el rango de edad fue de 32 a 85 años, con un promedio de 63.4 años (Tabla 2).

En relación al tiempo de evolución de la enfermedad antes de que el paciente se someta a cirugía se encontró que en Grupo 1 hubo una evolución clínica de 34 meses a diferencia del Grupo 2 en el cual la evolución clínica fue de 10 meses (Tabla 3).

Al analizar el cuadro clínico se encontró que dentro del Grupo 1 , 46 pacientes (38\%) presentaron lumbalgia, 45 pacientes (38\%) presentaron ciatalgia y 29 pacientes $(24 \%)$ presentaron claudicación. Dentro del Grupo 2, 21 pacientes (39\%) presentaron lumbalgia, 16 pacientes (30\%) presentaron ciatalgia y 17 pacientes (31\%) presentaron claudicación (Tabla 4).

Se tomó en cuenta la escala análoga de valoración del dolor (EVA), comparándola entre el preoperatorio y los controles postquirúrgicos (1, 3, 6 y 12 meses), encontrándose en el Grupo 1 un EVA pre quirúrgico de 8,56, postquirúrgico al mes de 1,3, a los 3 meses de 1,2, a los 6 meses de 1,2 y al año de 0,7. Mientras que en el Grupo 2 se encontró un EVA de 8,43 pre quirúrgico, postquirúrgico al mes de 1,8, a los 3 meses de 1,6, a los 6 meses de 1,4 y al año de 1,2 (Figura 7).

También se evaluó el grado de satisfacción del paciente correlacionándola con la integración del paciente a sus actividades diarias y de trabajo, dentro de esta variable se encontró en el Grupo 1 en el control postquirúrgico al mes un nivel de satisfacción de $86 \%$, a los 3 meses de 92\%, a los 6 meses de 91,4\% y al año de 94\%; y en el Grupo 2 al mes un nivel de satisfacción de $85 \%$, a los 3 meses de 98\%, a los 6 meses de 93,3\% y al año de 99\% (Figura 8).

Por último y como para tratar de describir las diferencias de los dos grupos se analizó la presencia o no de complicaciones en los dos grupos ya que ambos fueron sometidos al mismo procedimien-

Tabla 1. Comparativa en cuanto al género por grupos.

\begin{tabular}{c|c|c|c}
\hline & Numero & \multicolumn{2}{|c}{ Sexo } \\
\hline & & Maculino & Femenino \\
\hline Estenosis & 53 & $26(49 \%)$ & $27(51 \%)$ \\
\hline Listesis & 23 & $5(22 \%)$ & $18(78 \%)$ \\
\hline
\end{tabular}

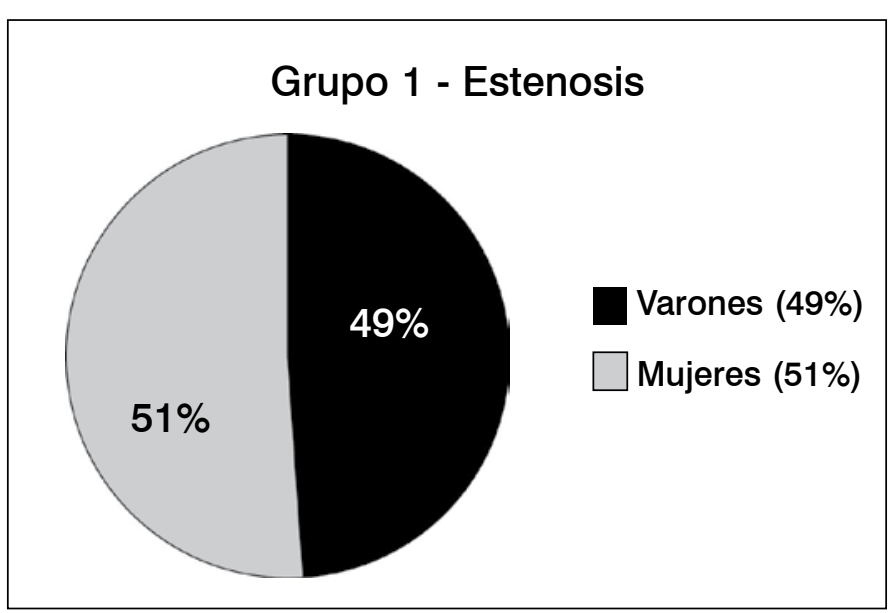

Figura 5. Comparación por genero en estenosis. to sin diferencias mayores, y se encontró que en el Grupo 1 se presentaron 4 complicaciones: 1 paciente presentó déficit neurológico el cual se recupero totalmente después de 18 meses de seguimiento, 2 pacientes con falla de implante, y 1 paciente con dehiscencia de herida. Dentro del Grupo 2 encontramos 2 complicaciones en total: 1 paciente con fistula de LCR y un paciente con infección de herida, ningún paciente requirió re intervención puesto que esas complicaciones se resolvieron con tratamiento conservador (Tabla 5).

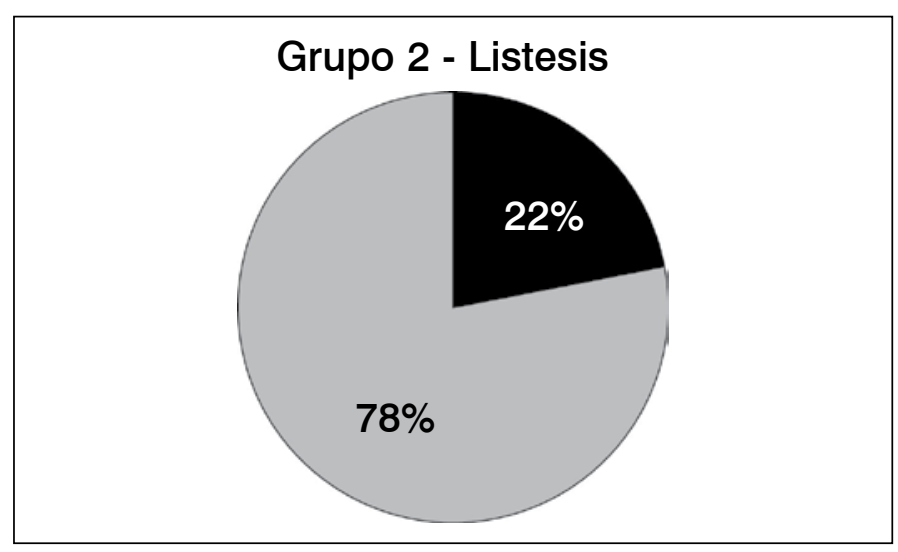

Figura 6. Comparación por genero en espondilolistesis.

Tabla 2. Comparativa en cuanto a la edad por grupos.

\begin{tabular}{c|c|c}
\hline \multicolumn{3}{|c}{ EDAD } \\
\hline & Grupo 1 - Estenosis & Grupo 2 - Listesis \\
\hline Rango (anõs) & $34-79$ & 32 - 85 \\
\hline Promedio & 56,8 & 63,4 \\
\hline
\end{tabular}

Tabla 3. Comparativa en cuanto al tiempo de evolución por grupos.

\begin{tabular}{c|c}
\hline \multicolumn{2}{c}{ Tiempo de Evolucion } \\
\hline Grupo 1 - Estenosis & 34 meses \\
\hline Grupo 2 - Listesis & 10 meses \\
\hline
\end{tabular}

Tabla 4. Comparativa en cuanto a la presentación clínica por grupos.

\begin{tabular}{c|c|c|c}
\hline \multicolumn{4}{|c}{ Clinica } \\
\hline & Lumbalgia & Ciatalgia & Claudicacion \\
\hline Grupo 1 - Estenosis & $46(38 \%)$ & $45(38 \%)$ & $29(24 \%)$ \\
\hline Grupo 2 - Listesis & $21(39 \%)$ & $16(30 \%)$ & $17(31 \%)$ \\
\hline Total & $67(39 \%)$ & $61(35 \%)$ & $46(26 \%)$ \\
\hline
\end{tabular}

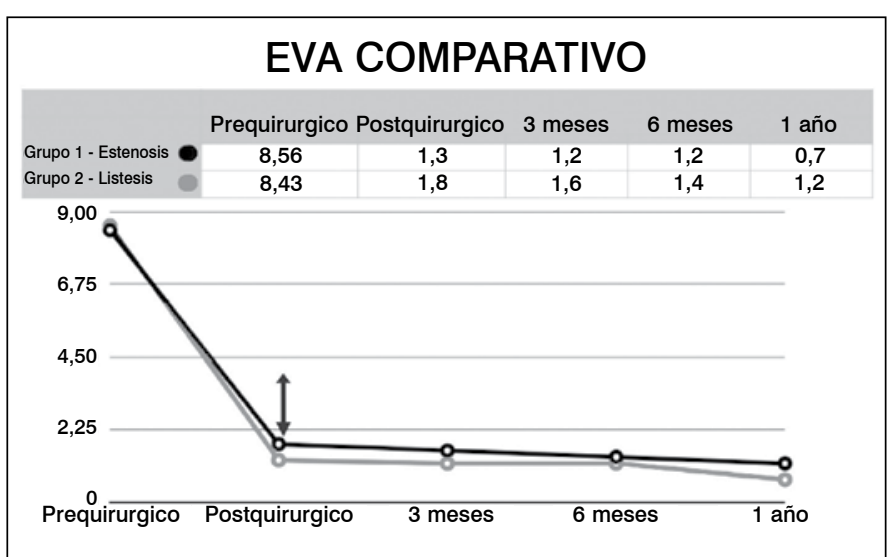

Figura 7. Comparación entre EVA pre-quirúrgico y postquirúrgico por grupos. 


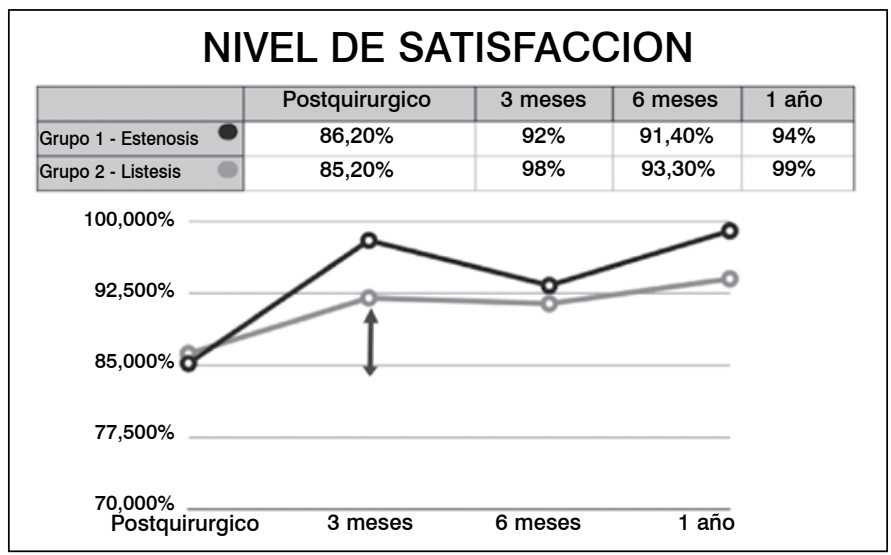

Figura 8. Comparación entre satisfacción pre-quirúrgico y postquirúrgico por grupos.

Tabla 5. Complicaciones por grupos.

\begin{tabular}{c|c|c}
\hline \multicolumn{3}{c}{ Complicaciones } \\
\hline & Grupo 1 - Estenosis & Grupo 2 - Listesis \\
\hline Deficit neurologico & 1 & - \\
\hline Fistula & - & 1 \\
\hline Falla de Implante & 2 & - \\
\hline Deshiscencia de herida & 1 & - \\
\hline Infección & - & 1 \\
\hline Total & 4 & 2 \\
\hline
\end{tabular}

\section{DISCUSION}

Al analizar la bibliografía, se evidencia claramente que a la estenosis degenerativa lumbar se la maneja como un síndrome en el cual se incluye a la estenosis degenerativa lumbar secundaria a espondilolistesis, pero dentro de nuestro análisis podemos demostrar que las dos patologías tienen unas características demográficas distintas en lo que respecta a edad y sexo, con un tiempo evolución diferentes y características fisiopatologícas con ciertas particularidades propias de cada una ${ }^{1,2,4,5}$.
Al analizar el manejo quirúrgico de nuestros dos grupos encontramos que Rillardon et al. ${ }^{8}$ en su publicación Long-term results of surgical treatment of lumbar spinal stenosis, y por otro lado e Iguchi et al. ${ }^{6}$ con su trabajo Minimum 10-year outcome of decompressive laminectomy for degenerative lumbar spinal stenosis, encontraron que existía un $70 \%$ de satisfacción al ser manejado quirúrgicamente el paciente, a diferencia de una muy baja satisfacción al ser manejado conservadoramente.

Postacchini et al. ${ }^{9}$ en su publicacion Surgical management of lumbar spinal stenosis, en la que realizó un estudio en 40 pacientes con estenosis de los cuales 16 eran secundario a espondilolistesis, con un seguimiento de 8,6 años, encontró que de los 16 pacientes 6 empeoraron su sintomatología y el grado de estenosis con el tiempo y los 6 coincidían en que no se les realizó artrodesis. En cambio a los 10 que si se les realizó artrodesis presentaron mejoría clínica mantenida.

Además Yukawa et al. ${ }^{10}$ nos hablan de que una descompresión amplia mejora los resultados clínicos a largo plazo y que el resultado clínico dependerá además del tiempo de evolución prequirúrgica de la sintomatología neurológica.

Gracias a esta amplia revisión bibliográfica se ha establecido el protocolo de manejo de los dos grupos en el estudio ${ }^{5,10-16}$, encontrán dose similares resultados en los dos grupos, en cuanto a la funcionalidad postquirúrgica, dolor postquirúrgico y complicaciones del procedimiento.

\section{CONCLUSIONES}

En cuanto a la distribución por géneros que en el Grupo 1 existe similitud entre sexo masculino y sexo femenino (49 y $51 \%$ respectivamente), pero en el Grupo 2 se encuentra una marcada predominancia del sexo femenino con un $78 \%$ y sobre el sexo masculino con un $22 \%$.

En relación a la edad podemos encontrar que dentro del Grupo 2 existe un promedio de 63,4 años de edad, casi 10 años más que en el Grupo 1 con un promedio de 56,8 años.

En relación a el tiempo de evolución del cuadro clínico vemos que este fue mucho mas rápido en el Grupo 2 (10 meses) en comparación con en el Grupo 1 que fue mas lento (34 meses), entendible por la mayor inestabilidad lumbar propia de la espondilolistesis.

\section{REFERENCIAS}

1. Spivak JM. Degenerative lumbar spinal stenosis. J Bone Joint Surg Am. 1998;80(7):1053-66.

2. Tan SB. Spinal canal stenosis. SingaporeMed J. 2003;44(4):168-9.

3. Arnoldi CC., Brodski AE., Lumbar Spinal Stenosis and Nerve root entrampment Syndrome, Definition and Classification. ClincOrthop. 1976; 115:4

4. NASS Clinical Guidelines Committee; Clinical Guidelines for Multidisciplinary Spine Care, Diagnosis and Treatment of Degenerative Lumbar Spinal Stenosis; January 2007 North American Spine Society.

5. Fritz JM, Delitto A, Welch WC, Erhard RE. Lumbar spinal stenosis: a review of current concepts in evaluation, management, and outcome measurements. ArchPhysMedRehabil. 1998;79(6):700-8

6. Iguchi T, Kurihara A, Nakayama J, Sato K, Kurosaka M, Yamasaki K. Minimum 10-year outcome of decompressive laminectomy for degenerative lumbar spinal stenosis. Spine (Phila Pa 1976). 2000;25(14):1754-9.

7. Koc Z, Ozcakir S, Sivrioglu K, Gurbet A, Kucukoglu S. Effectiveness of physical therapy and epidural steroid injections in lumbar spinal stenosis. Spine (Phila Pa 1976). 2009;34(10):985-9

8. Rillardon L, Guigui P, Veil-Picard A, Slulittel H, Deburge A. [Long-term results of surgical treatment of lumbar spinal stenosisl. Rev Chir Orthop Reparatrice Appar Mot. 2003;89(7):621-31.

9. Postacchini F, Cinotti G, Perugia D, Gumina S. The surgical treatment of central lumbar

stenosis. Multiple laminotomy compared with total laminectomy. J Bone Joint Surg Br. 1993;75(3):386-92

10. Yukawa Y, Lenke LG, Tenhula J, BridwellKH, RiewKD, Blanke K. A comprehensive study of patients with surgically treated lumbar spinal stenosis with neurogenic claudication. J BoneJointSurg Am. 2002:84-A(11):1954-9.

11. Atlas SJ, Keller RB, Wu YA, Deyo RA, Singer DE. Long-term outcomes of surgical and nonsurgical management of lumbar spinal stenosis: 8 to 10 year results from the maine lumbar spine study. Spine (Phila Pa 1976). 2005;30(8):936-43.

12. Lieberman I. Surgery reduced pain and disability in lumbar spinal stenosis better than nonoperative treatment. J Bone Joint Surg Am. 2007;89(8):1872.

13. Fu YS, Zeng BF, Xu JG. Long-term outcomes of two different decompressive techniques for lumbar spinal stenosis. Spine (Phila Pa 1976). 2008;33(5):514-8.

14. Westergaard L, Hauerberg J, Springborg JB. Outcome after surgical treatment for lumbar spinal stenosis: the lumbar extension test is not a predictive factor. Spine (Phila Pa 1976). 2009:34(25): E930-5

15. NASS Clinical Guidelines Committee; Clinical Guidelines for Multidisciplinary Spine Care, Diagnosis and Treatment of Degenerative Lumbar Spondylolisthesis; 2008 North American Spine Society

16. Jolles BM, Porchet $F$, Theumann N. Surgical treatment of lumbar spinal stenosis. Five-year follow-up. J Bone Joint Surg Br. 2001;83(7):949-53. 